DOI: http://dx.doi.org/10.12957/demetra.2014.10518

\title{
Estado nutricional de idosos, avaliados em domicílio, com dificuldade de acesso ao serviço de atenção básica
}

\author{
Nutritional status of elderly, valued at home, with limited access to primary care services
}

\author{
Regina do Rio Alvares' \\ Maria Regiane Trincaus ${ }^{2}$ \\ Carine Teles Sangaleti² \\ I Secretaria Municipal de Saúde de \\ Guarapuava-PR. \\ ${ }^{2}$ Departamento de Enfermagem da Universidade \\ Estadual do Centro-Oeste UNICENTRO. \\ Guarapuava-PR, Brasil. \\ Correspondência /Correspondence \\ Carine Teles Sangaleti \\ E-mail: sangaleti@usp.br
}

\section{Resumo}

Introdução: $\mathrm{O}$ aumento da expectativa de vida representa uma conquista social, mas pode ocasionar aumento no número de idosos dependentes, inseridos tanto no meio social quanto no familiar. A redução nos custos com assistência hospitalar e institucional é um dos motivos da permanência de idosos incapacitados em suas próprias casas, tornando o suporte domiciliar uma necessidade. O processo de avaliação da saúde de idosos engloba análises de aspectos clínicos e psicossociais, sendo o estado nutricional uma das condições clínicas de maior importância, devido a sua relação com a morbi-mortalidade dessa população. Objetivo: Verificar, em domicílio, o estado nutricional de idosos com dificuldade de acesso à unidade de saúde, por meio de diferentes instrumentos de avaliação nutricional. O estudo foi realizado com 18 idosos, de ambos os sexos, matriculados na Unidade de Saúde da Família - PSF Dourados, no Município de Guarapuava-PR, que recebiam visita regular de agente comunitário de saúde. Resultados: Grande parte dos idosos apresentava algum grau de desnutrição, e a avaliação por meio de medidas antropométricas foi mais acurada do que a realizada pela miniavaliação nutricional. Conclusão: Conclui-se que, independentemente do instrumento de análise utilizado, houve alta prevalência de desnutrição, e que a avaliação nutricional de idosos, realizada em domicílio, é potente ferramenta de avaliação e intervenção em seu estado nutricional.

Palavras-chave: Avaliação Nutricional. Idoso. Assistência Domiciliar. 


\section{Abstract}

Introduction: Increased life expectancy is a social achievement, but may rise the number of elderly dependents, both in the social environment and family environments. The reduction in the cost of hospital and institutional care is one reason for the permanence of the disabled elderly in their own homes, making the home support a necessity. The process of assessing the health of elderly encompasses analyzes of clinical and psychosocial aspects, and the nutritional status is of the clinical conditions of greater importance due to its relationship to morbidity and mortality in this population. Objective: To determine, in the household, the nutritional status of elderly with limited access to the facility through different nutritional evaluation tools. The study was conducted with 18 elderly men and women enrolled in the Family Health Unit - PSF Dourados, in the city of Guarapuava-PR, Brazil, who received regular visits from community health agents. Results: Many of the elderly had some degree of malnutrition, and evaluation using anthropometric measurements was more accurate than the one held by nutritional mini-evaluation. Conclusion: We conclude that, regardless of the instrument used for analysis, there was high prevalence of malnutrition, and nutritional assessment of the elderly, held at home, is powerful tool for evaluation and intervention in their nutritional status.

Key words: Nutritional Assessment. Elderly. Home Care.

\section{Introdução}

A população mundial está envelhecendo e, segundo a Organização Mundial da Saúde (OMS), o Brasil deverá ser o sexto país no mundo em número de idosos até o ano de 2025, com uma estimativa de 31,8 milhões. O segmento acima de 65 anos aumentará 8,9 vezes, e o acima de 80 anos, 15,6 vezes. Mantendo-se essa tendência demográfica, em números absolutos, o Brasil terá uma das maiores populações idosas na esfera mundial. ${ }^{1-4}$

Segundo a Pesquisa Nacional por Amostra de Domicílios (PNAD, 2009), entre os anos de 2008 e 2009 houve crescimento de 3,3\% na população com 60 anos ou mais, contra elevação de $1 \%$ no total da população residente no país. ${ }^{5}$ Para 2030, as projeções mostram um aumento na longevidade numa média nacional de 78,33 anos. ${ }^{6} \mathrm{Se}$, por um lado, o aumento da expectativa de vida representa uma conquista social, por outro pode ocasionar aumento no número de idosos dependentes inseridos tanto no meio social quanto no familiar. ${ }^{7,8}$ 
No Brasil, essa possibilidade constitui relevante desafio, visto que a rede de suporte social formada por serviços de saúde e assistência social, instituições formadoras de profissionais de saúde, dentre tantos outros, não se encontram preparadas para tal. Este despreparo ganha suporte, além das diferenças regionais e do quadro de desigualdade social brasileiro, no acelerado processo de transição demográfica, que se deu de forma muito mais rápida e intensa do que em países do dito Velho Mundo. ${ }^{9,10}$

Para Silva, Galera \& Moreno, ${ }^{8}$ a redução nos custos com assistência hospitalar e institucional é um dos motivos da permanência de idosos incapacitados em suas próprias casas, tanto no Brasil como em outros países, além de que a atual visão da assistência em saúde propõe que o idoso acometido de uma condição crônica e com incapacidades deva ser cuidado no ambiente onde sempre viveu. Neste contexto, coloca-se a necessidade de suporte ao idoso em domicílio.

A primeira referência em Assistência Domiciliar à Saúde (ADS) em sua forma organizada é o Dispensário de Boston, EUA, no ano de 1796, hoje, New England Medical Center. No Brasil, o primeiro sistema de ADS foi criado no Hospital do Servidor Público Estadual de São Paulo em 1967, tendo como principal objetivo reduzir os leitos ocupados. ${ }^{11}$

Destaca-se neste estudo a ótica da atenção à saúde, mais especificamente o componente nutricional, porque apesar de ser um processo natural, o envelhecimento envolve diversas modificações tanto anatômicas quanto funcionais que, embora variem de um indivíduo ao outro, podem repercutir na saúde e nutrição do idoso, predispondo-o ao surgimento de condições crônicas de saúde e possíveis sequelas debilitantes. ${ }^{8,12}$

Tais mudanças tornam mais complexa a assistência que deve ser dada a essa população, que carece de cuidados específicos e sistemáticos a suas necessidades ${ }^{13}$ devendo estar centrada na presença de profissionais de saúde capacitados no cuidado de idosos fragilizados, portadores de síndromes geriátricas e com perda da capacidade funcional, com ações de tratamento e reabilitação. ${ }^{14}$ Além disso, o processo de avaliação da saúde dos idosos engloba análises de aspectos clínicos e de variáveis psicossociais, sendo o estado nutricional uma das condições clínicas de maior importância, devido a sua relação com a morbi-mortalidade dessa população. ${ }^{15}$

Dentre as condições que interferem no estado nutricional do idoso, pode-se destacar, entre outras, a diminuição da capacidade funcional, da sensibilidade olfativa e gustativa, o uso de medicamentos, problemas psiquiátricos (demência, depressão, alcoolismo), sociais (isolamento, pobreza) e hábitos de vida (diminuição da atividade física, tabagismo, ingestão inadequada de alimentos). ${ }^{16}$ Observa-se também aumento da obesidade, principalmente a obesidade central, independente de gênero e idade, e de seus fatores de risco, como hereditariedade, hipertensão arterial sistêmica, resistência à insulina, diabetes mellitus, dislipidemias, além de maior incidência de doenças crônicas não transmissíveis ligadas à obesidade, como a aterosclerose e suas complicações. ${ }^{17}$ 
Dessa maneira, o objetivo deste estudo foi verificar, em domicílio, o estado nutricional de idosos com dificuldade de acesso à unidade de saúde, por meio de diferentes instrumentos de análise, com vistas a contribuir com informações atualizadas que possam trazer melhorias na qualidade de vida desses indivíduos.

\section{Material e métodos}

Estudo descritivo, transversal, que utilizou a metodologia de estudo de caso com análise quantitativa. A pesquisa foi aprovada pelo Comitê de Ética em Pesquisa (COMEP) da Universidade Estadual do Centro-Oeste (UNICENTRO), sob o parecer número 322/2011, sendo a participação de cada idoso autorizada pelo mesmo ou por responsável legal, mediante assinatura do Termo de Consentimento Livre e Esclarecido.

\section{Local do estudo}

Foi realizado em domicílios de idosos cadastrados na Unidade de Saúde da Família - PSF Dourados, no município de Guarapuava-PR. A unidade de saúde está localizada em um bairro da periferia, cuja fonte de renda principal é o trabalho informal e a renda média per capita da população inferior a um salário mínimo, ou seja, 622 reais no momento da pesquisa.

A área adstrita à unidade de saúde é composta por 3.600 pessoas, totalmente coberta, das quais 202 são idosos. A equipe é composta por um médico, uma enfermeira, uma técnica de enfermagem, uma auxiliar de enfermagem e seis agentes comunitários de saúde (ACS), num total de dez funcionários. A Unidade de Saúde da Família - PSF Dourados foi escolhida para a realização deste trabalho por estar situada na periferia da cidade, cuja população apresenta baixa renda familiar; por ser a área de abrangência da unidade de saúde muito esparsa e ter por isso áreas de grande dificuldade de acesso; por não possuir nutricionista em sua unidade e não utilizar nenhum dos parâmetros propostos para realizar uma avaliação do estado nutricional da população atendida.

\section{Casuística e métodos}

O estudo foi realizado com idosos de ambos os sexos, com idade igual ou superior a 60 anos, matriculados na unidade de Saúde da Família (USF), que recebiam visita regular de agente comunitário de saúde e apresentavam dificuldade física e/ou mental de acesso à unidade. A amostra de idosos foi constituída por 18 indivíduos identificados pelos ACS e que respondiam aos critérios de seleção. 
A coleta de dados foi realizada na presença do ACS por meio de questionário estruturado elaborado para este estudo, pela Miniavaliação Nutricional (MAN) para idosos e por avaliação antropométrica.

O questionário estruturado para este estudo foi elaborado para caracterização sociocultural dos idosos, para averiguação do estado civil, escolaridade, tipo de moradia, saneamento básico, renda familiar, participação em programas do governo e patologias autoreferidas ou referidas pelo familiar ou cuidador.

O teste MAN, que é composto de mensurações simples e de questões rápidas, podendo ser realizado em dez minutos, foi aplicado em todos os participantes deste estudo para avaliação do estado nutricional. O teste inclui as seguintes avaliações: avaliação antropométrica (peso, altura e perda de peso), avaliação global (seis perguntas relacionadas ao modo de vida, mobilidade e medicação), avaliação dietética (oito perguntas relacionadas ao número de refeições, ingestão de líquidos e alimentos e autonomia na alimentação) e avaliação subjetiva (autopercepção da saúde e nutrição). Consiste de questões que possuem pontuação de zero a três. Sua classificação é feita de acordo com o número de escore, sendo considerado um total de escore maior que 23,5, bem nutridos; entre 17 e 23,5, em risco de desnutrição; e menor que 17, desnutridos. ${ }^{15,18,19}$

A avaliação antropométrica foi realizada para estimar o estado nutricional dos idosos por meio da aferição do peso $(\mathrm{kg})$, estatura $(\mathrm{m})$, dobras cutâneas $(\mathrm{mm})$, circunferência do braço $(\mathrm{cm})$ e circunferência da panturrilha $(\mathrm{cm})$. As dobras cutâneas aferidas foram: dobra cutânea tricipital (DCT) e dobra cutânea subescapular (DCSe). Os indivíduos foram colocados, com roupas leves, em balança digital portátil da marca G-Tech®, com precisão de $100 \mathrm{~g}$ e capacidade para $150 \mathrm{~kg}$, para aferição do peso. A altura foi estimada utilizando-se a equação proposta por Chumlea ${ }^{20}$ por meio da medida da altura do joelho. A partir das medidas do peso e da estatura, também foi calculado o índice de massa corporal (IMC), utilizando-se os pontos de corte propostos pela Organização Panamericana da Saúde (OPAS) ${ }^{1}$ para idosos. Para obtenção da circunferência do braço (CB), foi utilizada uma fita métrica inextensível no ponto médio entre o acrômio e o olecrano, sendo os resultados comparados aos preconizados por Frisancho. ${ }^{21}$ A circunferência da panturrilha (CP) foi medida, utilizando-se uma fita métrica inextensível, com o indivíduo sentado, na área de maior diâmetro da panturrilha, tendo como pontos de corte valores menores que $31 \mathrm{~cm} .{ }^{22}$

A espessura das dobras cutâneas, conforme técnica preconizada por Heyward \& Stolarczyk, ${ }^{23}$ foi realizada em triplicata, do lado direito dos indivíduos, utilizando-se um adipômetro clínico da marca Cescorf ${ }^{\circledast}$. Foram mensuradas as dobras cutâneas triciptal e subescapular, calculando-se a média das três medidas. A DCT e DCSe foram avaliadas utilizando-se como referência as tabelas propostas por NHANES III ${ }^{15}$ e por Frisancho, ${ }^{21}$ respectivamente. Com as medidas da CB e DCT, foi calculada a circunferência muscular do braço $(\mathrm{CMB})$, por meio da fórmula $\mathrm{CMB}=\mathrm{CBcm}-(\Pi / 10$ x DCTmm), e os resultados comparados com as tabelas propostas por NHANES III.$^{15}$ 
A partir dos resultados das medidas antropométricas, foi realizado o diagnóstico do estado nutricional dos idosos, com base no IMC e segundo classificação de Blackburn \& Thomton, ${ }^{15}$ pela adequação da CB, DCT e CMB, em porcentagem, ou seja, a medida obtida dividida pelo valor do percentil 50 , na respectiva tabela, multiplicado por 100, sendo considerada desnutrição grave valor $<70 \%$; desnutrição moderada, $\geq 70 \%$ e $<80 \%$; desnutrição leve $\geq 80 \%$ e $<90 \%$; eutrofia $\geq 90 \%$ e $<110 \%$; sobrepeso $\geq 110 \%$ e $<120 \%$; obesidade valor $\geq 120 \%$.

Os resultados foram avaliados através de análise estatística descritiva simples através de frequências, médias e desvios-padrão, com auxílio do software Microsoft Excel ${ }^{\circledR}$. A classificação do estado nutricional foi realizada segundo os escores dos instrumentos de avaliação nutricional (conforme descrito anteriormente) e os resultados obtidos foram discutidos com base no embasamento teórico deste estudo e com o auxílio de outras pesquisas semelhantes na área.

\section{Resultados}

Neste estudo, foram avaliados 18 idosos, sendo a maioria $(77,8 \%)$ do sexo feminino. A idade dos idosos variou de 66 a 92 anos, sendo a média de 77,3 anos com desvio-padrão de 7,2 anos. A renda familiar média foi de 1.175,56 reais, com desvio-padrão de 567,08 reais, sendo que o número de moradores variou de dois a nove, com média de 4,28 e desvio-padrão de 1,99 moradores. A tabela 1 mostra a caracterização sociocultural dos idosos avaliados no estudo. Observa-se que grande parte dos avaliados eram viúvos ou solteiros (50\% e 27,8\%, respectivamente), e apresentavam baixa escolaridade, sendo que 33,3\% não eram alfabetizados e o restante não havia concluído o ensino fundamental. Destaca-se que quase a totalidade dos avaliados $(88,9 \%)$ não possuía saneamento básico e não participava de nenhum programa do governo (77,8\%). Hipertensão arterial sistêmica foi a patologia de maior frequência referida $(77,8 \%)$, seguida das doenças ósseas $(22,2 \%)$ e psiquiátricas $(22,2 \%)$. A alimentação dos idosos avaliados é preparada na maioria das vezes $(66,7 \%)$ por membros da família e em apenas 33,3\% dos casos os próprios idosos preparam sua própria alimentação. 
Tabela 1. Caracterização sociocultural dos idosos avaliados, em domicílio, pertencentes ao PSF- Dourados, Guarapuava-PR em 2012.

\begin{tabular}{|c|c|c|}
\hline Característica & $\mathrm{n}$ & $\%$ \\
\hline \multicolumn{3}{|l|}{ Sexo } \\
\hline Feminino & 14 & 77,8 \\
\hline Masculino & 4 & 22,2 \\
\hline \multicolumn{3}{|l|}{ Estado Civil } \\
\hline Viúvo & 9 & 50,0 \\
\hline Casado & 4 & 22,2 \\
\hline Solteiro & 5 & 27,8 \\
\hline \multicolumn{3}{|l|}{ Religião } \\
\hline Católica & 16 & 88,9 \\
\hline Evangélica & 2 & 11,1 \\
\hline \multicolumn{3}{|l|}{ Escolaridade } \\
\hline Fundamental incompleto & 12 & 66,7 \\
\hline Não alfabetizado & 6 & 33,3 \\
\hline \multicolumn{3}{|l|}{ Tipo de Moradia } \\
\hline Mista & 11 & 61,1 \\
\hline Alvenaria & 4 & 22,2 \\
\hline Madeira & 3 & 16,7 \\
\hline \multicolumn{3}{|l|}{ Saneamento Básico } \\
\hline Não & 16 & 88,9 \\
\hline Sim & 2 & 11,1 \\
\hline \multicolumn{3}{|l|}{ Participação em Programas do Governo } \\
\hline Nenhum & 14 & 77,8 \\
\hline Bolsa Família & 3 & 16,6 \\
\hline Auxílio Doença & 1 & 5,6 \\
\hline \multicolumn{3}{|l|}{ Patologias Referidas } \\
\hline Hipertensão Arterial Sistêmica & 14 & 77,8 \\
\hline Diabetes Mellitus & 2 & 11,1 \\
\hline Doenças Ósseas (artrose, osteoporose, reumatismo) & 4 & 22,2 \\
\hline Doenças Psiquiátricas (depressão, esquizofrenia, demência) & 4 & 22,2 \\
\hline Colesterolemia & 1 & 5,6 \\
\hline Efisema Pulmonar & 1 & 5,6 \\
\hline DPOC & 1 & 5,6 \\
\hline \multicolumn{3}{|l|}{ Preparo das refeições } \\
\hline Outras pessoas & 12 & 66,7 \\
\hline Própria pessoa & 6 & 33,3 \\
\hline
\end{tabular}

DPOC: Doença pulmonar obstrutiva crônica 
O estado nutricional, de acordo com a Miniavaliação Nutricional (MAN) para idosos, é mostrado na tabela 2. Não houve variação da média de escores entre os grupos. Observa-se que mais da metade dos idosos do sexo feminino (64,3\%) apresentava-se bem nutrida, mas destaca-se que $35,7 \%$ das mulheres apresentavam risco nutricional e desnutrição e que a maioria dos homens (75\%) encontrava-se em risco nutricional, segundo essa avaliação.

Tabela 2. Estado nutricional, segundo a MAN, de acordo com o gênero. Guarapuava-PR em 2012.

\begin{tabular}{cccccc}
\hline Classificação* & M de Score & $\begin{array}{c}\text { DP de } \\
\text { Score }\end{array}$ & $\begin{array}{c}\text { Bem } \\
\text { Nutrido }\end{array}$ & $\begin{array}{c}\text { Risco de } \\
\text { Desnutrição }\end{array}$ & Desnutrido \\
$\begin{array}{c}\text { Feminino } \\
(\mathrm{n}=4)\end{array}$ & 23,0 & 4,08 & $9(64,3 \%)$ & $3(21,4 \%)$ & $2(14,3 \%)$ \\
$\begin{array}{c}\text { Masculino } \\
(\mathrm{n}=4)\end{array}$ & 23,0 & 3,34 & $1(25 \%)$ & $3(75 \%)$ & 0 \\
\hline
\end{tabular}

*Escore de Indicação de desnutrição: $\geq 23,5$ pontos = Bem Nutrido; 17 a 23,5 pontos= Risco de Desnutrição; $<$ 17 Desnutrido

MAN - Mini avaliação nutricional

Na tabela 3, verifica-se o estado nutricional, segundo o IMC dos idosos avaliados, observando-se que a média não variou muito entre os sexos, ficando abaixo do recomendado pela OPAS (2005) ${ }^{1}$, ou seja, $23 \mathrm{~kg} / \mathrm{m}^{2}$. Destaca-se, ainda, que a maioria das mulheres $(64,3 \%)$ e dos homens $(75 \%)$ apresentou baixo-peso e que apenas 14,3\% das mulheres apresentaram sobrepeso.

Tabela 3. Estado nutricional, segundo IMC, de acordo com o gênero. Guarapuava-PR, 2012.

\begin{tabular}{|c|c|c|c|c|c|}
\hline $\begin{array}{c}\text { Classificação } \\
\text { de IMC** }\end{array}$ & M & DP & Baixo Peso & Eutrofia & Sobrepeso \\
\hline $\begin{array}{l}\text { Feminino } \\
\quad(n=14)\end{array}$ & 22,65 & 3,26 & $9(64,3 \%)$ & $3(21,4 \%)$ & $2(14,3 \%)$ \\
\hline $\begin{array}{l}\text { Masculino } \\
\qquad(\mathrm{n}=4)\end{array}$ & 20,48 & 3,75 & $3(75,0 \%)$ & $1(25,0 \%)$ & 0 \\
\hline
\end{tabular}

*Segundo OPAS, 20051. IMC $<23 \mathrm{~kg} / \mathrm{m}^{2}$ - Baixo Peso; IMC $\geq 23 \mathrm{~kg} / \mathrm{m}^{2} \mathrm{e}<28 \mathrm{~kg} / \mathrm{m}^{2}$ - Eutrofia;

IMC $\geq 28 \mathrm{~kg} / \mathrm{m}^{2}$ - Sobrepeso

IMC: Índice de massa corporal 
A avaliação antropométrica, utilizando vários instrumentos de análise, pode ser verificada na tabela 4. Observa-se que o resultado da avaliação, segundo a CB, mostrou que a totalidade dos idosos do sexo masculino (100\%) e 50\% do sexo feminino apresentavam algum grau de desnutrição. Quando o instrumento utilizado foi a DCT, verificou-se que mais da metade das mulheres $(64,3 \%)$ apresentavam algum grau de desnutrição e todos os homens (100\%) estavam desnutridos. Em relação à $\mathrm{CMB}$, a avaliação também mostrou que todos os idosos do sexo masculino apresentavam algum grau de desnutrição, mas que 64,3\% das mulheres encontravam-se eutróficas. Verificouse que a média da CP foi praticamente a mesma nos dois grupos avaliados, destacando-se que a metade do grupo dos homens apresentou algum grau de desnutrição.

Tabela 4. Estado nutricional de idosos, avaliados em domicílio por diferentes variáveis antropométricas, de acordo com o gênero. Guarapuava-PR, 2012.

\begin{tabular}{|c|c|c|c|c|c|}
\hline $\begin{array}{l}\text { Variável } \\
\text { Antropométrica }\end{array}$ & $\mathrm{M}$ & DP & Desnutrição* & Eutrofia & $\begin{array}{l}\text { Sobrepeso/ } \\
\text { Obesidade }\end{array}$ \\
\hline \multicolumn{6}{|l|}{ CB } \\
\hline Feminino $* *$ & 27,92 & 3,46 & $7(50 \%)$ & $5(35,7 \%)$ & $2(14,3 \%)$ \\
\hline Masculino*** & 25,85 & 1,12 & $4(100 \%)$ & 0 & 0 \\
\hline \multicolumn{6}{|l|}{ DCT } \\
\hline Feminino $^{* *}$ & 17,07 & 7,41 & $9(64,3 \%)$ & $2(14,3 \%)$ & $3(21,4 \%)$ \\
\hline Masculino $* * *$ & 7,25 & 1,26 & $4(100 \%)$ & 0 & 0 \\
\hline \multicolumn{6}{|l|}{ CMB } \\
\hline Feminino $* *$ & 22,51 & 2,05 & $3(21,4 \%)$ & $9(64,3 \%)$ & $2(14,3 \%)$ \\
\hline Masculino $* * *$ & 23,58 & 1,28 & $4(100 \%)$ & 0 & 0 \\
\hline \multicolumn{6}{|l|}{$\mathrm{CP}$} \\
\hline Feminino ${ }^{* *}$ & 33,07 & 3,47 & $4(28,6 \%)$ & $10(71,4 \%)$ & 0 \\
\hline Masculino $^{* * * *}$ & 32,65 & 3,62 & $2(50 \%)$ & $2(50 \%)$ & 0 \\
\hline
\end{tabular}

*Desnutrição (Grave/Moderada/Leve); **n=14; ***n=4; CB: circunferência do braço; DCT: dobra cutânea tricipital; CMB: circunferência muscular do braço; CP: circunferência da panturrilha 


\section{Discussão}

O envelhecimento populacional é uma realidade e os idosos que não estão em instituições encontram-se em casa. O cuidado integral prevê atenção com o estado nutricional e seus determinantes, o que torna a avaliação do estado nutricional de idosos importante ferramenta de trabalho das condições de saúde dessa população. Deve-se ressaltar, entretanto, que são escassos os estudos avaliando as condições nutricionais de idosos na atenção básica.

Distinguir desnutrição propriamente dita de desnutrição em consequência de uma doença é um desafio no prognóstico do idoso, pois não se tem ainda uma definição universalmente aceita para desnutrição. Praticamente todos os parâmetros da avaliação nutricional são afetados pelas alterações fisiológicas e patológicas. ${ }^{15}$

O presente estudo revelou que a totalidade dos idosos avaliados apresentava renda familiar abaixo de dois salários mínimos e baixa escolaridade, o que não surpreende, pois a USF está localizada numa região pobre da cidade. Nas doenças referidas, destaca-se a hipertensão arterial sistêmica, em cerca de dois terços dos idosos. Estudo realizado em Mato Grosso do Sul aponta resultados semelhantes em relação a renda familiar e escolaridade, além de alta prevalência de hipertensão como doença crônica. ${ }^{16}$

A MAN para idosos demonstrou o mesmo valor de escore médio para ambos os gêneros, e apesar de a maioria das mulheres estudadas se apresentarem bem nutridas, mais de $35 \%$ delas encontravam-se em risco nutricional ou desnutridas, e $75 \%$ dos homens em risco nutricional. Esses achados corroboram outros estudos realizados com idosos, nos quais o escore médio encontrado foi de 19,5 pontos, ${ }^{24}$ sendo que mais da metade dos avaliados encontrava-se em risco nutricional ou desnutridos. ${ }^{19,25}$ Em outro estudo, Felix e Souza, ${ }^{26}$ avaliando o risco nutricional de idosos institucionalizados, encontraram resultados semelhantes quando utilizaram a MAN para idosos como instrumento de avaliação, observando alta prevalência (70\%) de risco nutricional ou desnutrição nos homens.

A utilização do IMC para avaliação do estado nutricional de idosos apresenta dificuldades por não levar em consideração as alterações corporais típicas dessa faixa etária, como acúmulo de tecido adiposo e redução da massa magra. ${ }^{27}$ Entretanto, muitos estudos utilizam esse instrumento de análise em associação com outros instrumentos na avaliação do estado nutricional de idosos e adultos. ${ }^{2,19,26,28}$

Neste trabalho, os achados em relação ao IMC se mostraram mais sensíveis na detecção de mulheres com baixo-peso, diferentemente do que foi observado pela MAN. O IMC mostrou maior prevalência de mulheres (64,3\%) e de homens (75\%) com baixo-peso, apesar de 14,3\% das 
mulheres apresentarem sobrepeso. Resultados semelhantes foram encontrados no estudo realizado com idosos em Santa Catarina, onde foi constatada a maior prevalência (59,4\%) de baixo-peso nos homens. ${ }^{29}$ Andrade et al..${ }^{25}$ avaliando idosos em uma instituição de longa permanência, verificaram que quase a metade $(41,18 \%)$ estava eutrófica e 35,29\% apresentava sobrepeso, discordando dos resultados encontrados neste estudo. Lopes et al. ${ }^{2}$ também encontraram resultados discordantes ao verificarem maior prevalência de idosos com sobrepeso (38,7\%). A ocorrência de baixo-peso, assim como de sobrepeso, merece atenção, pois ambos os casos podem refletir riscos de doenças crônicas, tais como as cardiovasculares, a hipertensão arterial sistêmica e o diabetes mellitus, dentre as mais frequentes. ${ }^{26}$

A verificação do estado nutricional utilizando a CB irá refletir a redução da massa, pois esta medida representa a soma das áreas constituídas por tecidos gordurosos, muscular e ósseo do braço. Já a DCT é considerada um bom indicador de quantidade de tecido adiposo subcutâneo. Essas duas medidas combinadas, por meio de aplicação de fórmulas nos dá a CMB, medida que se correlaciona com a desnutrição protéica, refletindo o definhamento muscular. A CP é considerada medida sensível de massa muscular no idoso, indicando mudanças de massa livre de gordura que ocorrem com a idade e com a diminuição da atividade..$^{15}$

Os resultados encontrados com a utilização dessas medidas antropométricas demonstraram boa sensibilidade, pois se verificou que grande parte dos idosos apresentava algum grau de desnutrição, principalmente em relação ao grupo dos homens. Entretanto, deve-se ressaltar que em relação aos resultados encontrados de CMB e CP, pode-se dizer que os idosos avaliados não apresentaram grau elevado de desnutrição, pois a maioria das mulheres encontrava-se eutrófica, demonstrando que ainda há preservação da musculatura.

Gonçalves \& Mattos, ${ }^{30}$ analisando pacientes restritos ao domicílio, encontraram resultados semelhantes, verificando que em relação a CB e DCT, mais da metade dos idosos $(54 \%$ e $53 \%$, respectivamente) apresentou algum grau de desnutrição, e que $60 \%$ dos avaliados estavam eutróficos em relação à CMB. Outro estudo realizado no Distrito Federal com idosos institucionalizados encontrou resultados que corroboram os achados neste estudo em relação aos valores totais médios de $\mathrm{CP}(30,9 \pm 4,1)$ e em relação à $\mathrm{CB}$, constatando que 54,6\% das mulheres estavam desnutridas. ${ }^{26}$

O grau de dependência dos idosos é cada vez maior e esse idoso deve ser avaliado no contexto em que está inserido, com suas dificuldades e limitações, levando-se em conta as variações inerentes ao envelhecimento. 


\section{Considerações finais}

considerando o perfil de envelhecimento populacional, as condições sociais e a rede de suporte à saúde da região estudada, bem como os resultados sobre o estado nutricional da população avaliada, este estudo demonstrou que a avaliação nutricional de idosos, realizada em domicílio, é potente instrumento de avaliação e intervenção no estado nutricional dos mesmos e que a avaliação nutricional por meio de medidas antropométricas mostrou-se mais acurada que a avaliação realizada pela MAN.

Neste contexto, destaca-se a relevância do profissional nutricionista no âmbito da atenção básica, uma vez que a avaliação antropométrica é uma ferramenta específica do nutricionista. Além disso, no contexto da equipe de saúde, este estudo também demonstrou que a medida do IMC (aferido adequadamente) pode auxiliar na avaliação do risco nutrticional, mas essa medida não é valorizada ou sequer realizada.

Independentemente do parâmetro utilizado na avaliação do estado nutricional dos idosos, neste estudo detectou-se alta prevalência de desnutrição, que pode ser, talvez, atribuída à falta de conhecimento, preparo e orientação dos familiares e/ou cuidadores ou ainda pela falta de vínculo entre cuidador e idoso. Este resultado preocupa, pois não somente ele deve ser orientado em relação à qualidade da sua alimentação, mas também, e principalmente, os responsáveis pelos seus cuidados, para que possa ter melhor qualidade de vida.

Consideram-se como limitações neste estudo a utilização da MAN nas situações de déficits cognitivos e também a localização dos idosos a partir da indicação dos ACS, e não por busca ativa em todos os domicílios.

\section{Referências}

1. Organização Mundial da Saúde. Envelhecimento ativo: uma política de saúde. Brasília: OPAS; 2005.

2. Lopes AF, Braga CP, Boliani E, Almeida FQA. Perfil antropométrico e alimentar dos participantes do Programa Universidade Aberta à Terceira Idade (UNATI) do Instituto de Biociências de Botucatu/ SP. Rev. Ciênc. Ext. 2010; 6(1):1-13.

3. Costa MFBNA, Ciosak SI. Atenção integral na saúde do idoso no Programa Saúde da Família: visão dos profissionais de saúde. Rev. Esc. Enferm. 2010; 44:(2):437-44.

4. Cavalcanti CL, Gonçalves MCR, Cavalcanti AL, Costa SFG, Asciuti LSR. Programa de intervenção nutricional associado à atividade física: discurso de idosas obesas. Ciência \& Saúde Coletiva 2011; 16(5):2383-90.

5. Instituto Brasileiro de Geografia e Estatística. Pesquisa Nacional por Amostra de Domicílios. Síntese de indicadores. Rio de Janeiro: IBGE; 2009. 
6. Instituto Brasileiro de Geografia e Estatística. Indicadores Sociodemográficos. Prospectivos para o Brasil 1991-2030. Rio de Janeiro: IBGE; 2006.

7. Chaimowicz F. A saúde dos idosos brasileiros às vésperas do século XXI: problemas, projeções e alternativas. Rev. Saúde Pública 1997; 31(2):184-200.

8. Silva L, Galera SAF, Moreno V. Encontrando-se em casa: uma proposta domiciliar para famílias de idosos dependentes. Acta Paul. Enferm. 2007; 20(4):397-403.

9. Wong LLR, Carvalho JÁ. O rápido processo de envelhecimento populacional do Brasil: sérios desafios para as políticas públicas. Rev. Bras. Est. Popul. 2006; 23(1):5-26.

10. Nogueira SL, Geraldo JM, Machado J, Ribeiro RCL. Distribuição espacial e crescimento da população idosa nas capitais brasileiras de 1980 a 2006: um estudo ecológico. Rev. Bras. Estud. Popul. 2008; 25(1):195-98.

11. Amaral NN, Cunha MCB, Ladronici RHDD, Oliveira ASB, Gabbai AA. Assistência Domiciliar à Saúde (Home Health Care): sua História e sua Relevância para o Sistema de Saúde Atual. Rev. Neurociências 2001; 9(3):111-17.

12. Campos MTFS, Monteiro JBR, Ornelas APRC. Fatores que afetam o consumo alimentar e a nutrição do idoso. Rev. Nut. 2000; 13(3):157-65.

13. Rosa MRQP, Patrício ZM, Silvério MR, Rumel D. Motivos que levaram idosos a buscar a atenção em uma unidade básica de saúde. Rev. Latino Am. Enfermagem 2009; 17(5): 670-76.

14. Veras R. Envelhecimento populacional contemporâneo: demandas, desafios e inovações. Rev. Saúde Pública 2009; 43(3):548-54.

15. Cuppari L. Guia de nutrição: nutrição clínica no adulto. 2. ed. Barueri, SP: Manole; 2005.

16. Alvarenga MRM, Oliveira MAC, Faccenda O, Amendola F. Avaliação do risco nutricional em idosos atendidos por Equipes de Saúde da Família. Rev. Esc. Enferm. USP 2010; 44(4):1046-51.

17. Dantas AMC, Soares EA, Frank AA. A obesidade como precursora de aterosclerose encontrada em indivíduos da terceira idade. Revista Brasileira de Obesidade, Nutrição e Emagrecimento 2007; 1(3):60-71.

18. McGee M, Jensen GL. Mini Nutritional Assessment (MNA): research and practice in the elderly. Am. J. Clin. Nutr. 2000; 71(1):158.

19. Campanella LCA, Farias MB, Breitkopf'T, Almeida CB, Mendes L, Fenilli M et al. Relação entre padrão alimentar e estado nutricional de idosos hospitalizados. Rev. Bras. Nutr. Clin. 2007; 22(2):100-6.

20. Chumlea, WC, Roche AF, Mukherjee D. Nutritional assessment of the elderly through anthropometry. Columbus, Ohio: Ross Laboratories; 1987. 45 p.

21. Frisancho AR. New norms of upper limb fat and muscle areas for assessment of nutritional status. Am. J. Clin. Nutr. 1981; 34(1):2540-5.

22. World Health Organization. Physical status: the use and interpretation of antropometry. Geneva: WHO; 1995. Who Thechnical Report Series, 854. 
23. Heyward VH, Stolarczyk LM. Avaliação da composição corporal aplicada. São Paulo: Manole; 2005.

24. Paula HAA, Oliveira FCE, São José JFB, Gomide CI, Alfenas RCG. Avaliação do estado nutricional de pacientes geriátricos. Rev. Bras. Nutri. Clin. 2007; 22(4):280-5.

25. Andrade DDG, Fonseca SS, Stracieri APM. Mini Avaliação Nutricional, avaliação da capacidade cognitiva e funcional de idosos em uma instituição de longa permanência no Município de Ipatinga, MG. Nutri Gerais Rev. Dig. Nutr. [online]. 2009; 3(5):428-43.

26. Felix LN, Souza EMT. Avaliação nutricional de idosos em uma instituição por diferentes instrumentos. Rev. Nutr. 2009; 22(4):571-80.

27. Bicudo-Salomão A, Aguilar-Nascimento JE, Caporossi C. Risco nutricional em cirurgia avaliado pelo índice de massa corporal ajustado ou não para pacientes idosos. Arq. Gastroenterol. 2006; 43(3):219-23.

28. Barbosa AR, Souza JMP, Lebrão ML, Marucci MFN. Estado nutricional e desempenho motor de idosos de São Paulo. Rev. Assoc. Med. Bras. 2007; 53(1):75-9.

29. Ruen MS, Moreira EAM, Calvo MCM, Lobo AS. Avaliação do estado nutricional de idosos intitucionalizados. Rev. Nutr. 2008; 21(3):303-10.

30. Gonçalves SP, Matos KM. Perfil nutricional de pacientes restritos ao domicílio na região oeste de Santa Maria, RS. Disc. Scientia 2008; 9(1):135-47.

Recebido: $15 / 4 / 2014$

Revisado: 03/7/2014

Aprovado: 02/8/2014 